\title{
Beam Shaping and Power Handling of an Spatial Light Modulator System for Laser Induced Periodic Surface Structuring Texturation
}

\author{
Julien Dupuy*, Hendrik Von Horsten, Marc Lamblin and Yves Hernandez \\ Multitel a.s.b.l, Parc Initialis, Mons, BE 7000 \\ ${ }^{*}$ Corresponding author's e-mail:dupuy@multitel.be
}

\begin{abstract}
Laser Induced Periodic Surface Structuring (LIPSS) is a powerful tool for creating submicrometric features on large surfaces in an up-scalable and industrial way. In order to control the process and reach a sufficient uniformity for some applications a careful attention must be paid to the control of the laser beam parameters like its polarization, stability and shape. A uniform beam shape is mandatory in some cases. Although some demonstrations of beam shaping with high power handling optical components have been made, there is not much reported regarding the use of SLM (Spatial Light Modulator) for beam shaping of ultrashort pulses. In the current work we show the possibility to use SLM with different type of laser sources, including a femtosecond system delivering up to $1 \mathrm{~mJ}$ per pulses.
\end{abstract}

DOI: $10.2961 / \mathrm{jlmn} .2020 .03 .2012$

Keywords: SLM, LIPSS, beam shaping.

\section{Introduction}

Various micro-machining processes based on lasers require a total control of the beam shape for accurate and regular patterning. The Gaussian beam shape exhibited by the laser source must be, most of the time, converted into a homogeneous top-hat shape. Such shape permits a uniform distribution of light onto the sample. This is particularly the case for Laser Induced Periodic Surface Structuring (LIPSS) process [1]. The ripples generated by LIPSS and in particular their periodicity, strongly depend on the laser fluence.

LIPSS had found lot of scientific and industrial applications, such as antireflection [2], colorization [3], hydrophobic surface [4] and biological devices [5], so the importance of fabrication and modulation of LIPSS on large surface area is a popular topic.

The orientation of the LIPSS patterns depends on the laser beam polarization [6]. Then, the orientation of the polarization can be also required for reaching given functionalities and even polarization modification during the process can be desired for some specific applications. Polarization control can be achieved easily for linear polarization orientation, with classical, high optical damage threshold optics, but one should just pay attention to the used of multiple elements together: SLM and polarization control for instance.

Beam shaping can be achieved with classical diffractive optical elements in order for instance to get a uniform tophat shape [7]. This is a robust and cost-effective solution that suffers only one drawback which is its nonreconfigurable mode of operation. Regarding this aspect, spatial light modulators provide much more flexibility but are more limited in terms of power and energy handling.
In this work we present the use of a commercial SLM beam shaping system with femtosecond pulses for the generation of LIPSS. We will discuss the initial performances of the component and its optimization with adequate thermal management [8] and then the application of the device for LIPSS generation on stainless steel.

\section{Initial performance of the SLM device}

We used a spatial light modulator from the company HoloEye. The PLUTO-2 device is a reflective LCOS PAN (Parallel Aligned Nematic)-SLM and allows for phase modulation. It has an array of $1920 \times 1080$ pixels. The Pixel pitch of the modulator is $8 \mu \mathrm{m}$. The device frame rate is $60 \mathrm{~Hz}$ and, to address the pixels, a HDTV 8 bits grayscale image must be uploaded via a HDMI display port. The fill factor of the modulator is $93 \%$.

Our first observations with this module were the following:

- The output beam shaping result is strongly dependant on the quality of the input beam. One needs to maximize the pixels surface illumination with a good fitting input beam

- The device operation showed power limitations: the SLM worked properly up to $7 \mathrm{~W}$ in nanosecond pulsed regime (100ns, $1 \mathrm{~mJ}$ pulses).

- The device output power is sensitive to input polarization state: Due to the polarization properties of the nematic liquid-crystal SLM, an input laser beam linearly polarized needs to have the good angle to maximize the reflection of the SLM. The use of a half wave plate in front of the device to align the polarization is required. 


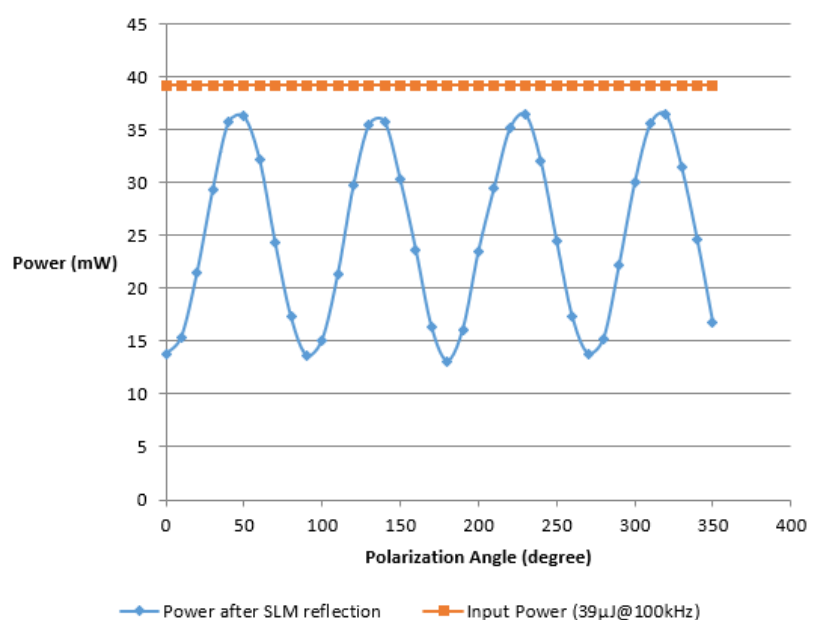

Fig. 1 Monitoring of the optical power variation at the output of the SLM in function of the polarization input angle.

Depending of the polarization angle of the laser input beam, the measured power losses of the SLM system vary from 7 to $65 \%$.

\section{SLM improvements on power handling}

We designed a specific holder for the SLM device. This holder permits a water cooling of the SLM plate. The SLM is mounted directly against a copper chamber with water circulation (Fig.2 (1)). A small frame (Fig.2 (2)) fix the SLM panel. An Aluminium base (Fig.2 (3)) was made to hold the copper chamber and fix mechanically the device on a breadboard for experiments.
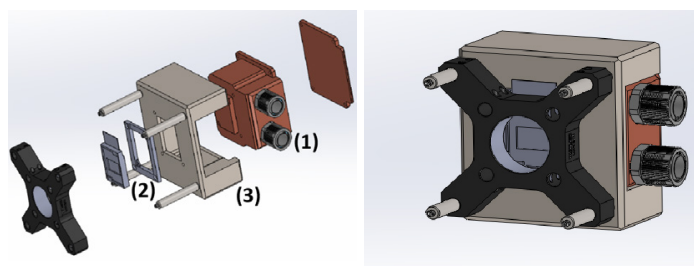

Fig. 2 CAD of the SLM water cooling holder.

The base and the copper chamber contain also embedded thermistors to monitor the temperature.

Based on this holder, we were able to carry new power handling tests, with three different IR lasers: a continuous wave (CW) laser operating @1064nm, a nanosecond fiber laser which can deliver $20 \mathrm{~W} 1 \mathrm{~mJ} 200 \mathrm{~ns}$ pulses, and an USP (Ultra-short Pulses) laser@1030nm which can deliver 4 Watts average power with $\mathrm{mJ}$ pulses.

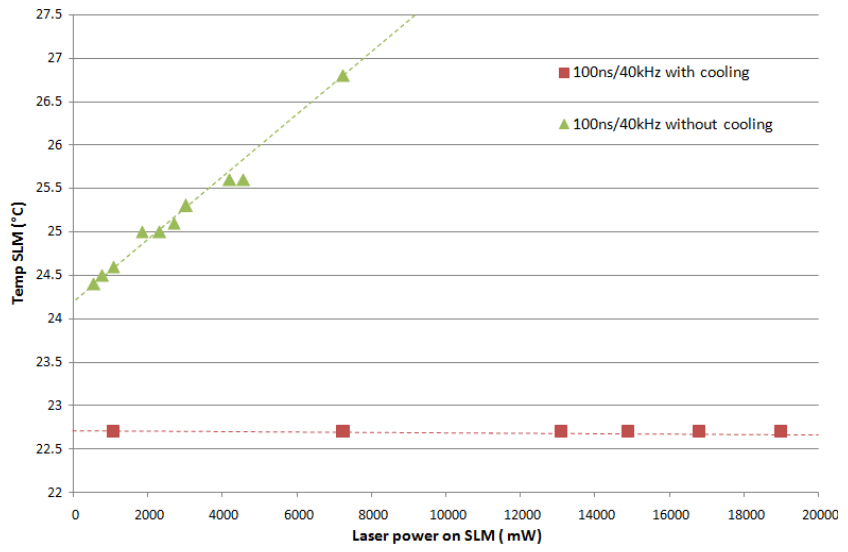

Fig. 3 Evolution of the SLM temperature in function of the ns laser input average power with and without water cooling.

In $\mathrm{CW}$ mode the device was tested with more than $100 \mathrm{~W}$ at $1064 \mathrm{~nm}$, but for pulsed applications, with high peak powers, one needs to be careful especially if the beam diameter is very small and average power density is quite high $>50 \mathrm{~W} / \mathrm{cm}^{2}$. Based on this water-cooling holder we were able to demonstrate power handling larger than $20 \mathrm{~W}$ average power in nanosecond regime and up to $1 \mathrm{~mJ}$ with $500 \mathrm{fs}$ pulses ( $2 \mathrm{GW}$ peak power) without observed degradation.

\section{Implementation of the SLM on a laser microm- achining bench}

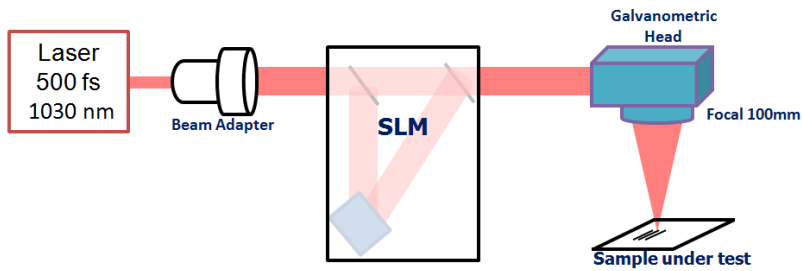

Fig. 4 Laser Setup for testing the SLM module.

The SLM module was inserted on a laser micromachining setup. A Beam Expender is mandatory in order to adapt the initial Gaussian beam diameter to the maximum surface of the LCOS-PAN panel. We worked here with a $8 \mathrm{~mm}$ beam diameter $\left(2 \omega_{0}\right)$ at the entrance.

We placed two flip mirrors on the beam path in order to easily switch between the pristine Gaussian beam of the femtosecond laser, and the beam after shaping by the SLM, for a better comparison of the micromachining results.

In front of the SLM device, we used a half-wave plate, held in a rotation mount, to control the incident polarization of the laser beam on the SLM panel and maximise its reflectance (Fig1). Before the output mirror (second flip mirror) we placed another half-wave plate in a rotation mount as well to change the output polarization for LIPSS process control.

After the SLM, we have a $4 \mathrm{f}$ configuration consisting of two plano-convex fused silica lenses, with an AR-coating and $100 \mathrm{~mm}$ focal length, with a diaphragm (which acts like a high-pass filter on the optical FFT generated by the SLM). 


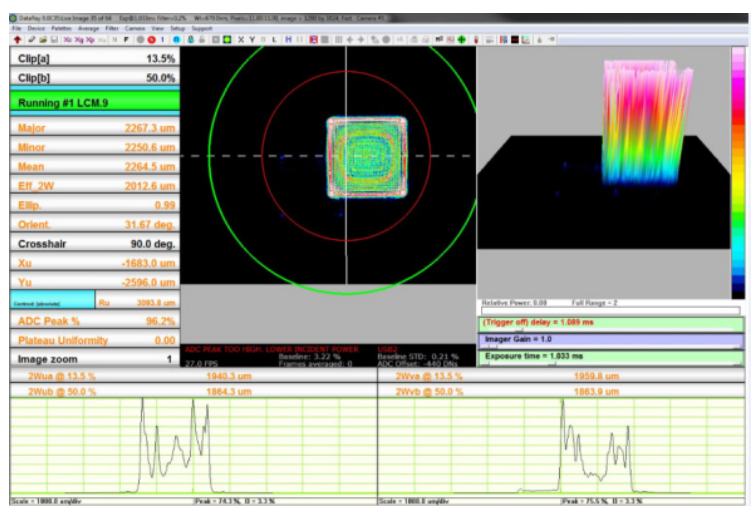

Fig. 5 Beam shaping after the SLM, pictures taken just after the SLM and 4F filter. Laser source used : 1030nm 500fs $100 \mathrm{kHz} 40 \mu \mathrm{J}$

We controlled the beam shape and size with a Dataray beam-profiling camera. We used the software provided by the SLM manufacturer Holoeye to generate patterns. It has already an embedded function to convert Gaussian to square top-hat beam. Depending on the phase map algorithm or the picture sent to the computing unit of the SLM, we produced a square shape but the energy distribution inside was unperfect (no flat top). We could make some improvements with a dynamic corrective feedback loop that precisely analyzes the shape, diameter and defects of the Gaussian beam at the input of SLM (wavefront correction) [9]

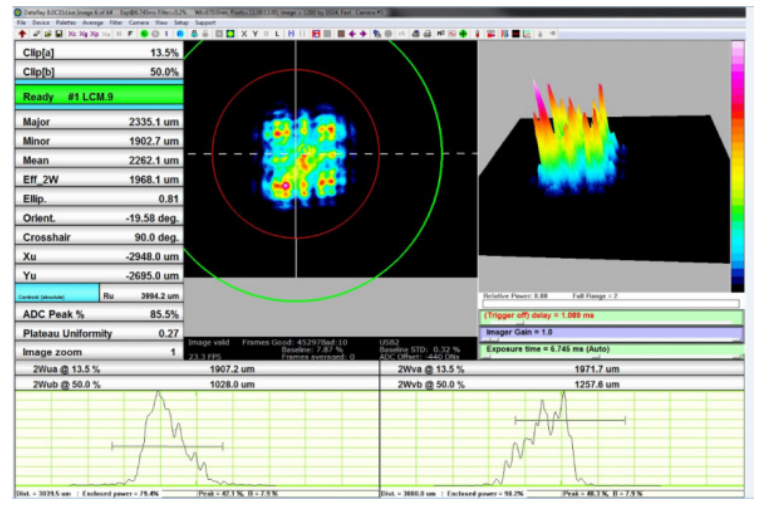

Fig. 6 Beam shaping with SLM : shape after a focal lens f100mm; picture taken after the galvanometric head at $+50 \mathrm{~mm}$ after the focal point.

The figure 6 gives us an evaluation of the shape and energy distribution at the vicinity of the focal point. We placed the Dataray camera directly onto the beam. This method made it impossible to capture the exact focal point because of the energy density and the camera resolution.

\section{Tests}

\subsection{Non-overlapping laser shots and scanning lines with the SLM module}

First, we tested a unitary laser shot on the materials. For this experiment, the laser frequency was fixed at $1 \mathrm{kHz}$ and the maximum energy per pulse was near $1 \mathrm{~mJ}$. This experience produced pulses with high and uniform energy deposited on the sample, but we were also far higher than the energy density required to produce LIPSS and only ablation appeared.

Then we fixed the repetition rate of the laser at $100 \mathrm{kHz}$ in order to have sufficient energy per pulse and also for easy testing with low scanning speeds (below $500 \mathrm{~mm} / \mathrm{s}$ ).
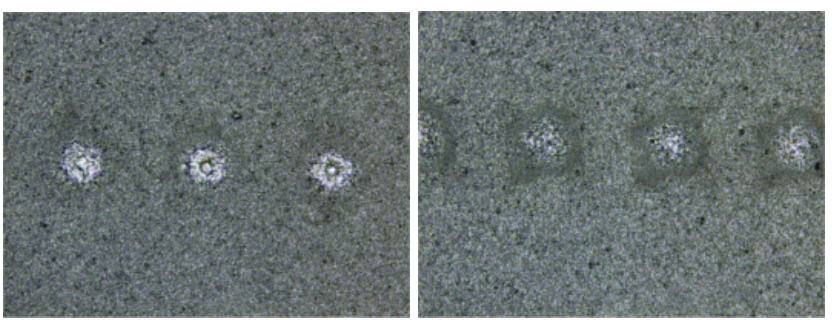

Fig. 7 Laser impacts on a Silicon Wafer V250mm/s $100 \mathrm{kHz}$ (Left) Gaussian Beam (Right) SLM beam shaped
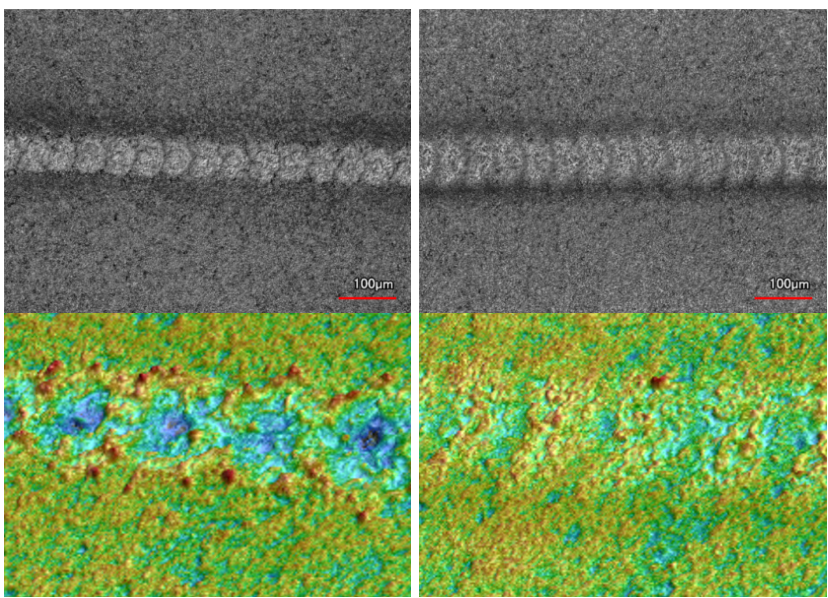

Fig. 8 Laser scribed lines on a Silicon Wafer V50mm/s $100 \mathrm{kHz}$ (Left) Gaussian Beam, (Right) SLM beam shaped

The focused laser spot size is smaller with a Gaussian beam than the square top hat shape $(\sim 30 \mu \mathrm{m}$ vs $55 \mu \mathrm{m}$, estimated and measured on the sample).

For all the tests performed, with unitary laser shots or with scribed lines, we can clearly see a better energy distribution on the material using the SLM module with a square top hat shape, compared with the initial Gaussian laser beam of the laser.

\subsection{LIPSS texturation tests with the SLM Module}

We worked mainly with inox316L polished sheets of $0.25 \mathrm{~mm}$ thickness. The LIPSS was produced with a laser beam emitting at $1030 \mathrm{~nm}$ and delivering $500 \mathrm{fs}$ pulses at a repetition rate of $100 \mathrm{kHz}$ and an average power of $3.3 \mathrm{~W}$.

This parameter gives us a single shot fluence near $1 \mathrm{~J} / \mathrm{cm}^{2}$. The scanning speed was $250 \mathrm{~mm} / \mathrm{s}$, corresponding to an approximate number of pulses of 22 and a cumulative fluence of $24 \mathrm{~J} / \mathrm{cm}^{2}$. These values are comparable with similar experiments from the literature [10] on stainless steel. 


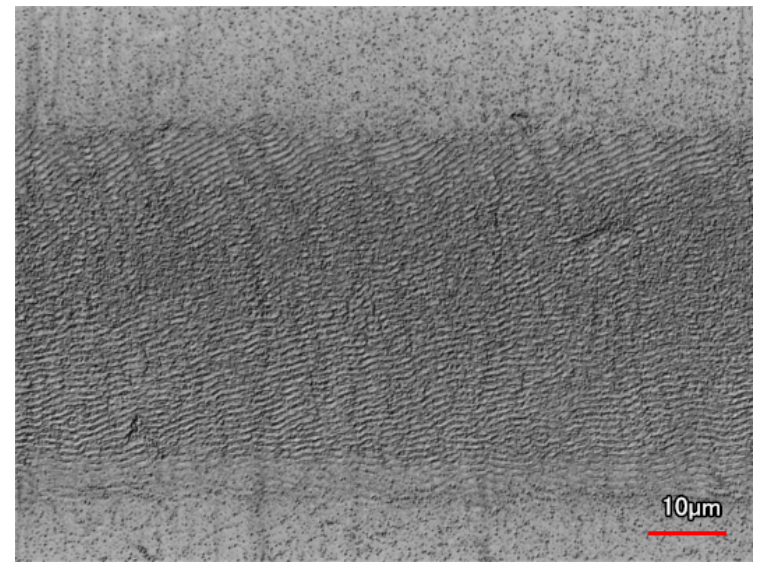

Fig. 9 LIPSS performed on INOX 316L with the SLM Module coupled with the $500 \mathrm{fs}$ laser.

On figure 9, we can clearly see homogenous LIPSS with a well-defined border between the initial surface and the irradiated one. We have obtained a period of $930 \mathrm{~nm}$ with peak to valley height $945 \mathrm{~nm} \Delta 120 \mathrm{~nm}$ on all the $48 \mu \mathrm{m}$ scribed laser line width.

\section{Conclusion}

We tested the possibility of using a thermalized Spatial Light Modulator for beam shaping with three different lasers:

- a nanosecond laser, up to $20 \mathrm{~W}$ and $1 \mathrm{~mJ}$,

- a CW laser up to $100 \mathrm{~W}$

- a femtosecond laser delivering up to $1 \mathrm{~mJ}$ (2 GW peak power).

We still don't know the laser power handling limit under femtosecond regime for the water cooled SLM as we were limited by the available average power and energy from our laser sources.

The global system permits both polarization and beam shape control for producing homogeneous LIPSS patterns in a dynamically reconfigurable way. Mainly used to shape the laser in a square flat top-hat shape, the SLM also permits a flexible and adaptive laser beam shaping. Although other methods can provide homogenous LIPSS with a welldefined border, even fabricated by Gaussian beam [11 12], The use of this SLM device to produce regular ripples patterns is more ready to be integrated on industrial micromachining machine with high power USP laser.

This system was applied to stainless steel texturing. Regular LIPSS could be generated at a scanning speed of 250 $\mathrm{mm} / \mathrm{s}$.

\section{Acknowledgments}

This work was carried out within the frame of the project LASER4SURF, funding from the European Union's Horizon 2020 research and innovation program under grant agreement No 768636 .

\section{References}

[1] G.R.B.E. Römer, A.J. Huis in't Veld, J. Meijer, and M.N.W. Groenendijkb: CIRP Annals, 58, (2009) 201.

[2] A.Y. Vorobyev and C. Guo: Opt. Express, 19, (2011) 1031.

[3] A.Y. Vorobyev and C. Guo: Appl. Phys. Lett., 92, (2008) 041914.
[4] B. Wu, M. Zhoua, J. Li, X. Ye, G. Li, and L. Cai: Appl. Surf. Sci., 256, (2009) 61.

[5] T.S. Drakakis: App. Phy. Lett., 89, (2006) 144108.

[6] U. Hermens, M. Pothen, K. Win, K. Arntz, and F. Klocke: Opt. Lasers Engin., 101, (2018) 44.

[7] G. Zhu, D. Whitehead, W. Perrie, O.J. Allegre, V. Olle, Q. Li, Y. Tang, K. Dawson, Y. Jin, S.P. Edwardson, L. Li, and G. Dearden: J. Phys. D: Appl. Phys., 51 (2018) 095603.

[8] R. J Beck, J. P Parry, W. N MacPherson, A. Waddie, N. J Weston, J. D Shephard, and D. P Hand: Opt. Express, 18, (2018) 17059.

[9] S. Li, Z. Lu, P. Du, Y. Wang, L. Ding, and X. Yan: Proc. SPIE, Vol. 10710, (2018) 107103Q.

[10] T. Jwad, P. Penchev, V. Nasrollahi, and S. Dimov: App. Surf. Sci., 453, (2018) 449.

[11]L. Wang, Q. Chen, X. Cao, R. Buividas, X. Wang, S. Juodkazis, and H. Sun: Light Sci. Appl., 6, (2017) 17112.

[12] J. Huang, L. Jiang, X. Li, A. Wong, Z. Wang, Q. Wang, J. Hu, L. Qu, T. Cui, and Y. Lu: Nanophotonics, 8, (2019) 869.

(Received: June 24, 2020, Accepted: October 30, 2020) 